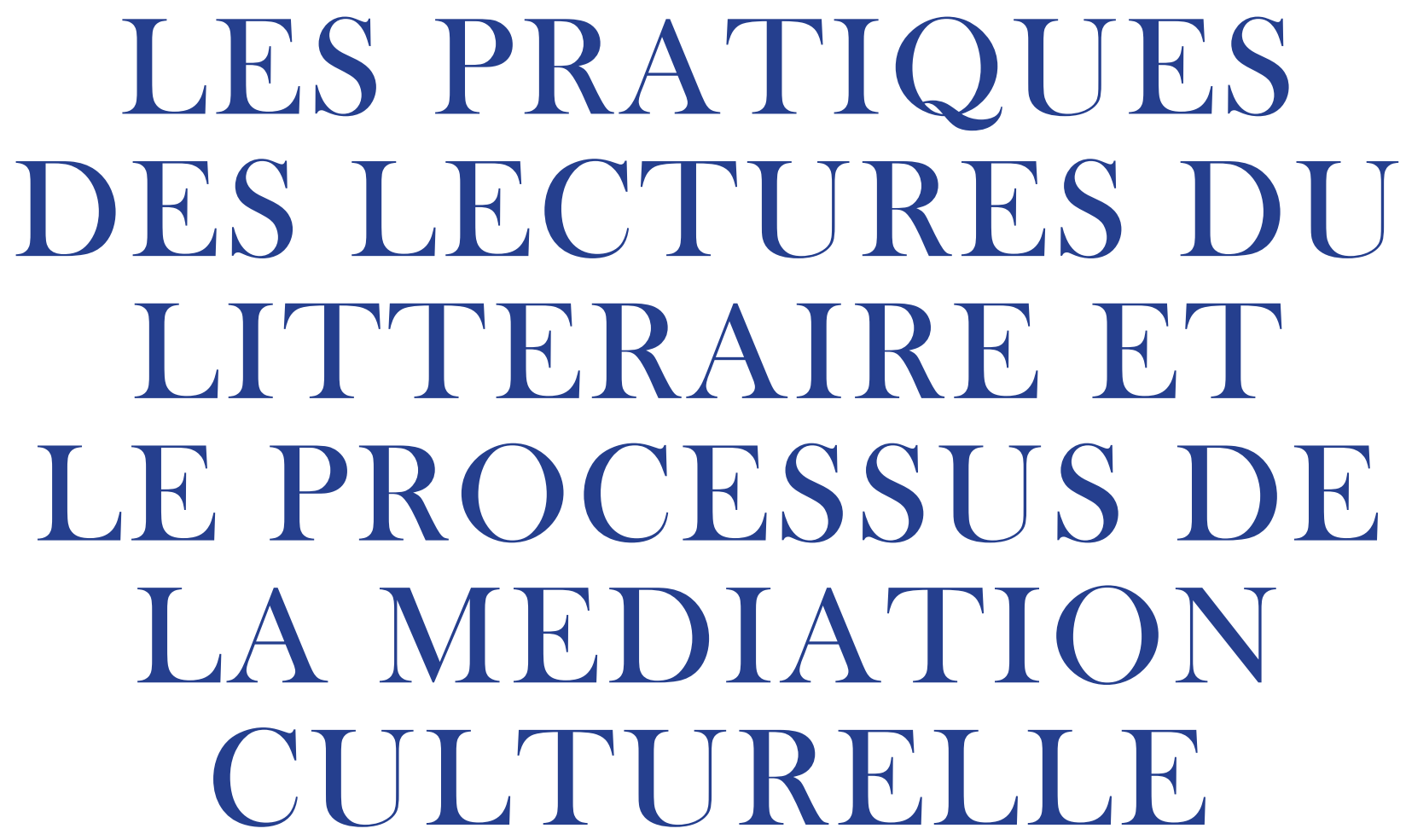

AS PRÁTICAS DE LEITURAS LITERÁRIAS E OS PROCESSOS DE MEDIAÇÃO CULTURAL

\title{
Entrevista com Anne Kupiec
}

(Doutora em Sociologia e Professora do Centro de Ciência Sociais da Universiadde de Paris VII - Diderot)

ntre os anos 2013 e 2014 tive a alegria de conviver com pesquisadores do Laboratoire de Changement Social et Politique - LCSP - da Universidade Paris Diderot, entre eles Anne Kupiec. Em conjunto, desenvolvemos a pesquisa intitulada 'A literatura como prática social', que teve como objetivo aprofundar as abordagens teórico-metodológicas relacionadas à leitura do texto literário. Durante 11 meses investigamos questões relativas à formação de leitores na França e no Brasil. A França, país considerado por nós, brasileiros, como modelo em políticas públicas relacionadas à leitura, vive hoje problemas que não se assemelham aos nossos (que ainda estão relacionados ao acesso aos livros), mas que revelam algumas fragilidade nesse campo. A última pesquisa feita acerca das práticas culturais dos franceses revelou 
que o número de livros lidos vem diminuindo inclusive entre os jovens que finalizaram curso superior. Anne Kupiec, na entrevista a seguir, aborda a leitura do literário do ponto de vista da sociologia da leitura, respondendo questões como: A França é ainda um país de leitores? Como se comporta o mercado editorial hoje? Como que a escola francesa lida com a literatura? Quais são as condições necessárias para a formação de leitores?

Neitzel - Comment percevez-vous les pratiques des lectures en France, aujourd'hui? Êtes vous optimiste quant aux pratiques de lecture de la jeunesse? La France est-elle encore un pays de lecteurs?

Kupiec - L'enquête sur les Pratiques culturelles des Français réalisée, à intervalles réguliers, par le département des études, de la prospective et des statistiques du ministère de la Culture depuis 1973, montre depuis une quinzaine d'années, un tassement de l'intensité de la lecture de livres et de presse notamment parmi les classes d'âge les plus jeunes (l'enquête est soumise à des personnes âgées de 15 ans et plus). Or, les classes d'âge les plus jeunes sont, aujourd'hui, proportionnellement plus diplômées que leurs aînées en raison de la relative démocratisation scolaire. Cette démocratisation s'est accompagnée d'une diversification des filières tant dans l'enseignement secondaire (bacs général, technologique et professionnel que dans l'enseignement supérieur (introduction et développement des cursus professionnels et professionnalisants qui concernent aujourd'hui environ la moitié des étudiants). Dès lors, les contenus des manuels scolaires ont évolué et le recours à la lecture s'est fait moins impératif notamment dans le domaine de la littérature.

Autrement dit, les critères d'âge et de diplômes qui constituaient des facteurs favorables à une forte intensité de lecture ne sont plus aussi significatifs que dans le passé. L'on constate que cette évolution se poursuit et rien ne laisse entrevoir, du moins pour l'instant, un changement significatif dans les années à venir. Les "gros ${ }^{2} »$ lecteurs de livres sont aujourd'hui de moins en moins nombreux. Ce que l'on constate également, c'est la diminution des tirages dans le domaine de la production éditoriale en sciences humaines et sociale. En revanche, il est remarquable d'observer l'augmentation de la production de livres pratiques, catégorie de livres qui rassemble le plus fort taux de lecteurs.

Neitzel - Quelle différence faites-vous entre les livres de littérature qui sont publiés aujourd'hui en France et ceux du passé?

Kupiec - Il est probable que l'âge du roman classique fut le XIX ${ }^{\text {e }}$ siècle. Cette époque est révolue. Le roman s'est transformé en France, mais pas seulement, 
au cours du XXe siècle, que l'on songe à Kafka ou à Joyce, au «nouveau roman» (Nathalie Sarraute, Alain Robbe-Grillet...) ou à Queneau. Naturellement, les conditions socio-historiques ne sont pas sans effets sur la production romanesque. Des auteurs ont cependant fait part de leurs préoccupations ${ }^{3}$ face aux changements présents. Mais peut-être faudrait-il considérer la place croissante prise par la documentation, par l'information, toujours renouvelées, susceptibles d'induire un mode de lecture tout autre que celui requis par le roman.

Neitzel - Au Brésil, on discute beaucoup sur la nécessité de la « lecture plaisir » à l'école, dont nous parle Roland Barthes (Au plaisir du texte) pour la formation d'un gros lecteur. Comment l'école française rend-elle en compte I' enseignement de la littérature, surtout aux premiers niveaux (école élémentaire et college)? Est-que la littérature est utilisée seulement comme soutien pédagogique ou est elle respectée comme l'art?

Kupiec - La littérature est associée à l'apprentissage du français à l'école élémentaire. Désormais, la littérature pour la jeunesse a sa place à l'école: des romans, des albums sont travaillés en classe, mais les enfants ont aussi la possibilité de découvrir d'autres livres dans la bibliothèque scolaire. Au collège, la littérature est abordée dans les cours de français. La lecture suivie, autrement dit la lecture complète d'un ouvrage, est présente et tente de développer «le plaisir de lire». Tant à l'école qu'au collège, une place très importante est accordée à la littérature dite «de jeunesse». Il s'agit-là d'ouvrages spécifiquement écrits pour telle ou telle tranche d'âge quoique les contes, y compris les plus anciens, soient toujours présents. Cette production, devenue considérable, donne accès à des textes supposés convenir parfaitement à des écoliers et des collégiens. L'on peut s'interroger sur cette production destinée à accroître le plaisir de la lecture des plus jeunes, mais qui les tient parfois à l'écart d'un corpus littéraire qui était commun à plusieurs générations et sur lequel il était possible d'échanger au sein de la famille. Les objectifs et les livres lus au lycée sont évidemment différents : il s'agit notamment, indiquent les instructions ministérielles, d'enrichir la culture littéraire des élèves, de former leur jugement et de développer une conscience esthétique. Il reste que, dans le cadre scolaire, la lecture est prescrite.

Neitzel - Martine Poulain dans un entretien publié dans Les mutations de la lecture (2012, p. 72) affirme au sujet de la lécture en France, que "le plus préoccupant est sans doute que la lecture de livres est également en 


\section{diminution dans les catégories diplômées, supérieures". Que pensez-vous de ce phénomène de désaffection pour la lectures des livres?}

Kupiec - Ainsi que je le disais précédemment, c'est un des constats que l'on peut faire à la lecture de l'enquête sur les Pratiques culturelles des Français. Il est certain que la diversification de l'offre audiovisuelle entendue au sens large (multiplication des chaînes de télévision, offre variée de jeux vidéo, accès à des textes, des images et des musiques grâce à internet) multiplie les choix de loisirs qui peuvent aussi apparaître plus attrayants et, le cas échéant, entrer en concurrence avec la lecture. L'on constate aussi que la notion de «légitimité culturelle», chère à Bourdieu, a évolué tant dans les déclarations que dans les pratiques. Au début des années 1990, Olivier Donnat, maître d'oeuvre de l'enquête déjà citée, évoquait l'éclectisme dont feraient preuve les catégories les plus diplômées (Les Français face à la culture. De l'exclusion à l'éclectisme, La Découverte, 1994). Quelques année plus tard, Bernard Lahire (La Culture des individus. Dissonances culturelles et distinction de soi, La Découverte, 2004) soulignait les dissonances culturelles qui concerneraient près de $80 \%$ de la population. Ainsi les pratiques de lecture, parce que représentatives d'une légitimité culturelle, auraient perdu leur attrait. Mais l'on pourrait aussi faire I'hypothèse que la norme de légitimité s'est affaissée, ce qui expliquerait l'écart entre les déclarants d'il y a quarante ans et ceux d'aujourd'hui.

Contrairement à ce qui se passait au XIXe siècle, l'accès à la maîtrise de la lecture peut désormais être acquis par tous grâce à la scolarisation obligatoire. Si la pratique de la lecture a pu être associée, dans le passé, à I' émancipation politique, cette époque est révolue : l'accès à l'imprimé (livre et presse) est aisé, l'imprimé est abondant et ne constitue plus un enjeu politique comme ce fut le cas. Cette situation peut être observée en France et dans la plupart des pays occidentaux.

\section{Neitzel - Selon vous, quelles sont les conditions idéales pour être un gros lecteur, surtout quand on pense aux enfants et à la jeunesse?}

Kupiec - Bien des parents de jeunes enfants considèrent qu'une grande familiarité avec le livre, quasiment dès la naissance, fera de leur progéniture des lecteurs assidus. Sans vouloir s'arrêter sur les motifs qui poussent des parents à fournir des livres à leurs enfants, et cela en fonction de leur âge, selon la segmentation des publics imposée par les éditeurs de livres pour la jeunesse, il importe de souligner que le livre, devenu si accessible, ne relève plus de l'interdit, de la transgression qui, elle aussi, est formatrice pour les plus jeunes. 
L'on méconnaît relativement les effets d'un usage intense des écrans d'ordinateur par les plus jeunes. Certains observateurs estiment que cet usage des écrans, y compris pour y lire des textes, ne sont pas sans effets sur les modalités de connaissance et d'intellection. On peut supposer que ce type de lecture sur écran, qui ne concerne pas les romans, conduise à des modification qui affecteraient la lecture du livre. A cet égard, il sera intéressant de prêter attention aux modes de lecture sur liseuse si ce dispositif se développe dans les années qui viennent. Il convient aussi de ne pas négliger les effets des productions de masse et des innovations des industries culturelles. Je pense notamment aux contenus accessibles sur un «smartphone».

Bref, les conditions idéales pour créer de gros lecteurs ne sont pas simples à déterminer dans ce contexte actuel, mais peut-être ces conditions idéales n'existent-elles pas? Michel Peroni, dans une étude qui s'intéressait aux adultes (Histoires de lire, BPI, Centre Georges Pompidou, 1995), démontrait qu'en termes de pratique de lecture rien n'était jamais ni complètement perdu ni complètement acquis. Le parcours biographique et les manières dont chacun se représente le monde (y compris symboliquement) sont susceptibles d'avoir un effet, ou non, sur l'intensité de la lecture.

En outre, il importe aussi de prendre en compte le contexte social et politique. Le lien qui a pu être noué pendant des siècles entre lecture et émancipation, lecture et citoyenneté semble aujourd'hui, tout du moins dans notre aire géographique ${ }^{4}$, largement distendu. Les événements à venir modifieront peut-être cette situation. La lecture n'est plus associée à un horizon politique comme elle a pu l'être, ici, dans le passé, alors qu'elle l'est, aujourd'hui, ailleurs.

NOTAS

1 Entrevista realizada pela Professora Adair de Aguiar Neitzel com a Professora Anne Kupiec.

2 Les «gros» lecteurs sont ceux qui déclarent lire plus de vingt livres par an ; ils représentaient $16 \%$ des personnes interrogées lors de la dernière enquête en 2008.

3 Milan Kundera, L'art du roman, Gallimard, 1986, Les testaments trahis, Gallimard, 1993; Alain Nadaud, Malaise dans la littérature, Champ Vallon, 1993; Tzvetan Todorov, La littérature en péril, Flammarion, 2007; Pierre Jourde, La littérature sans estomac, L'Esprit des péninsules, 2001.

4 Michèle Petit analyse ainnsi une situation tout autre en Amérique Latine: L'Art de lire ou comment résister à l'adversité, Belin, 2008. 